\title{
Vacuum Evaporation of Pure Metals
}

\author{
JAFAR SAFARIAN and THORVALD A. ENGH
}

Theories on the evaporation of pure substances are reviewed and applied to study vacuum evaporation of pure metals. It is shown that there is good agreement between different theories for weak evaporation, whereas there are differences under intensive evaporation conditions. For weak evaporation, the evaporation coefficient in Hertz-Knudsen equation is 1.66. Vapor velocity as a function of the pressure is calculated applying several theories. If a condensing surface is less than one collision length from the evaporating surface, the Hertz-Knudsen equation applies. For a case where the condensing surface is not close to the evaporating surface, a pressure criterion for intensive evaporation is introduced, called the effective vacuum pressure, $p_{\text {eff. }}$ It is a fraction of the vapor pressure of the pure metal. The vacuum evaporation rate should not be affected by pressure changes below $p_{\text {eff, }}$, so that in lower pressures below $p_{\text {eff, }}$, the evaporation flux is constant and equal to a fraction of the maximum evaporation flux given by Hertz-Knudsen equation as $0.844 \dot{n}_{\text {Max }}$. Experimental data on the evaporation of liquid and solid metals are included.

DOI: $10.1007 / \mathrm{s} 11661-012-1464-2$

(C) The Author(s) 2012. This article is published with open access at Springerlink.com

\section{INTRODUCTION}

THE kinetics of materials' evaporation in vacuum is important in many areas such as materials' processing and materials' application in vacuum and space systems. In metallurgical processes, the kinetics of materials' evaporation is important when mass transfer occurs from a condensed phase to a gas phase. Developing basic knowledge of the evaporation kinetics of pure metals is crucially important. The distillation of metals and vacuum refining for eliminating the impurities are typical processes in which the application of low pressures is the key process. Studying the mass transport phenomena in such processes, where the evaporation is taking place from a multicomponent condensed phase, requires basic knowledge of the evaporation of the single component systems. For instance, in the vacuum refining of metals, useful information about the mass transfer coefficient of the volatile impurity in the gas phase can be determined from the gas velocity above the melt, which mainly consists of the solvent metal vapor, and is relatively close to the vapor velocity of the pure solvent metal. The vacuum removal of phosphorus $(\mathrm{P})$ from liquid silicon $(\mathrm{Si})$ is a typical case in which very low concentrations of $\mathrm{P}$ such as $10 \mathrm{ppmw}$ are eliminated from $\mathrm{Si}$ to achieve the concentrations required for the fabrication of silicon solar cells, i.e., below 0.1 ppmw..$^{[1]}$ Considering the above points, the vacuum evaporation of pure elements is studied in this paper as follows.

JAFAR SAFARIAN, Researcher, and THORVALD A. ENGH, Professor, are with the Department of Materials Science and Engineering, Norwegian University of Science and Technology (NTNU), Alfred Getz Vei 2, 7491 Trondheim, Norway. Contact e-mail: Jafar.Safarian@material.ntnu.no

Manuscript submitted June 5, 2012.

Article published online October 17, 2012

\section{KINETIC THEORIES OF EVAPORATION}

In a perfect vacuum condition, the maximum molar flux of substance Me from the condensed form to its gaseous form is expressed by the Hertz-Knudsen equation. ${ }^{[2]}$

$$
\dot{n}_{\text {Max }}=\frac{p^{\circ}}{\sqrt{2 \pi M R T}}
$$

where $M$ is the molecular weight, $R$ is the universal gas constant, and $T$ is the absolute temperature at the evaporating surface. $p^{\circ}$ is the standard vapor pressure of the substance Me, which is a function of the absolute temperature ${ }^{[3]}$

$$
\log p^{\circ}=\frac{A}{T}+B \log T+C T+D
$$

The coefficients $A, B, C$, and $D$ are constants. When perfect vacuum is not used, the net flux of Me from the condense phase to its vapor is expressed as

$$
\dot{n}_{\text {evap. }}=\frac{p^{\circ}-p}{\sqrt{2 \pi M R T}}
$$

where $p$ is the pressure above the condensed phase. Equation [3] is based on considering a certain distribution function of velocity for the gas particles (full range Maxwellian), where the particles do not interact with each other, but move freely between collisions. ${ }^{[4]}$ Schrage ${ }^{[4]}$ introduced a simple correction to take the continuum into account and argued that the Maxwellian due to the mass movement of the vapor must be shifted by the mean velocity of the gas. According to Schrage, Eq. [3] becomes

$$
\dot{n}_{\text {evap. }}=\frac{p^{\circ}}{\sqrt{2 \pi M R T}}-\frac{\Gamma p}{\sqrt{2 \pi M R T_{\mathrm{g}}}}
$$


where $T_{\mathrm{g}}$ is the vapor temperature far from the evaporation interface. $\Gamma$ recognizes the effect of the vapor mass flow $\left(v_{\mathrm{g}}\right)$

$$
\Gamma=\exp \left(-S^{2}\right)-\sqrt{\pi} S(1-\operatorname{erf}(S))
$$

where

$$
S=\sqrt{\frac{M}{2 R T_{\mathrm{g}}}} v_{\mathrm{g}}
$$

The dimensionless parameter $S$ is called external speed ratio. Based on several investigations on the kinetics of evaporation, ${ }^{[5-8]}$ it is generally believed that the velocity distribution function at the vapor-liquid interface may deviate from a Maxwellian, and a transition to continuum flow occurs in a layer, which is known as the Knudsen layer. Thus, the vapor space may be divided into two regions: a Knudsen layer and an outer region with gas dynamic flow. The Knudsen layer is only a few mean-free paths in thickness. For instance, an extent of 20 mean-free paths has been obtained from the Monte Carlo simulation. ${ }^{[5]}$ The Knudsen layer expands with increasing bulk gas velocity away from the interface surface. For vapor velocities greater than the velocity of sound, expansion occurs outside the Knudsen layer in the continuum flow. Ytrehus and Østmo ${ }^{[9]}$ studied the basic fluid flow and thermodynamic aspects of interphasial processes in single-component condensed-vapor systems employing kinetic theory. According to them, the net evaporated mass flux is given as

$$
\dot{n}_{\text {evap. }}=\frac{p^{\circ}}{\sqrt{2 \pi M R T}}-\frac{\Gamma \beta p}{\sqrt{2 \pi M R T_{\mathrm{g}}}}
$$

where $\beta$ is a non-equilibrium backscattering factor ${ }^{[9]}$ and is a function of the vapor mass flow

$$
\beta=\frac{2\left(2 S^{2}+1\right) \sqrt{\frac{T_{\mathrm{g}}}{T}}-2 \sqrt{\pi} S}{\Gamma+\sqrt{\frac{T_{\mathrm{g}}}{T}}\left(\left(2 S^{2}+1\right)(1-\operatorname{erf} S)-\frac{2 S}{\sqrt{\pi}} \exp \left(-S^{2}\right)\right)}
$$

The ratio of $\frac{T_{\mathrm{g}}}{T}$ is the ratio of the gas temperature over the temperature at the liquid-gas interface. According to several studies, ${ }^{[7-11]}$ this temperature ratio can be expressed as

$$
\sqrt{\frac{T_{\mathrm{g}}}{T}}=-\frac{\sqrt{\pi}}{8} S+\sqrt{1+\frac{\pi}{64} S^{2}}
$$

In order to evaluate the coefficients $\Gamma$ and $\beta$ in Eqs. [4] and [7], these parameters were calculated against $S$ and the results are shown in Figure 1. It is observed that $\Gamma$ decreases with increasing vapor velocity. In contrast, $\beta$ increases with increasing $S$, and this increase is significant at $S$ values larger than $S \approx 0.5$. The change of $\Gamma \beta$ against $S$ is, however, different and it decreases with increasing of the gas phase velocity to a minimum of $\Gamma \beta=0.53$ around $S=0.55$, and then it increases with increasing of the gas velocity.

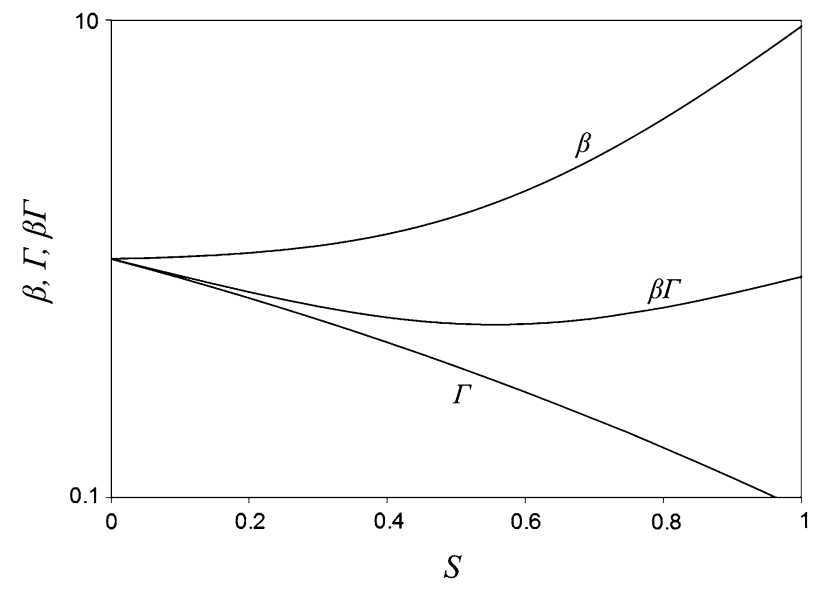

Fig. 1-Relationship between the coefficients in Eqs. [4] and [7] with the dimensionless external speed ratio.

Theories of evaporation and condensation, which are applied to a wide range of non-equilibrium conditions such as the above-mentioned studies, are mostly based on assumptions regarding the form of the velocity distribution function of vapor molecules at the surface. According to Algie,${ }^{[12]}$ however, the distribution function describing vapor molecules moving away from the surface is not the same as for the emission of molecules from the condensed phase. Algie ${ }^{[12]}$ extended Crout's approach $^{[13]}$ for evaporation (where vapor near the evaporating surface is considered as non-isotropic) and obtained an expression for the $\frac{n_{\text {evap }}}{\dot{n}_{\text {Max }}}$, where an evaporation coefficient $\alpha$ is taken into account

$$
\Phi_{\text {Algie }}=\frac{\dot{n}_{\text {evap. }}}{\dot{n}_{\text {Max }}}=\frac{\alpha}{1+\alpha\left(\frac{\theta}{2 S}-1\right)}
$$

where

$$
\theta=\frac{\exp \left(-S^{2}\right)}{\sqrt{\pi}}+S(1+\operatorname{erf}(S))
$$

According to Algie ${ }^{[12]}$ of the molecules which would leave the surface in the absence of surface constraints, only the fraction $\alpha$ actually leaves the surface. Algie does not seem to specify how to determine $\alpha$.

In spite of extensive studies on evaporation, the Hertz-Knudsen expression is still widely used due to its simple form, in particular for practical applications. Considering the temperature difference on the evaporation interface and the bulk gas phase, and introducing an apparent evaporation coefficient ( $\eta$ ), Eq. [3] becomes

$$
\dot{n}_{\text {evap. }}=\eta\left(\frac{p^{\circ}}{\sqrt{2 \pi M R T}}-\frac{p}{\sqrt{2 \pi M R T_{\mathrm{g}}}}\right)
$$

For weak evaporation $\left(p / p^{\circ} \rightarrow 1\right)$, the $S$ value is small and negligible, and the Schrage's expression reduces to Eq. [12] with $\eta=2 .^{[12,14]}$ This differs by a factor of 2 from the Hertz-Knudsen equation.[3] Evaporation in a semi-infinite space has been studied through the development of a linear kinetic theory of condensation and 
evaporation at small Knudsen numbers using the Navier-Stokes and Fourier equations by Muratova and Labuntsov. ${ }^{[15]}$ They obtained an evaporation coefficient for weak evaporation conditions $\eta=\frac{\alpha}{1-0.4 \alpha}$, where $\alpha$ is a constant smaller than unity. A similar expression for weak evaporation was also obtained later with another approach by Labuntsov and Kryukov. ${ }^{[16]}$ Considering the evaporation coefficient $\alpha$ as unity, $\eta=1.667$ is obtained. Algie ${ }^{[12]}$ indicated that for weak evaporation, $\eta=\frac{\alpha}{1-0.355 \%}$, and this gives $\eta=1.55$ for $\alpha=1$. Koffman et al. ${ }^{[14]}$ applied the kinetic theory approach with continuum flow in gas between a hot liquid surface and a cold liquid surface. They also challenged the physics of the Knudsen layer. For weak evaporation, they calculated $\eta=1.665$, which is in agreement with the above-mentioned studies. Ytrehus and Østmo obtained $\eta=1.668$ for weak evaporation, in which the results are linearized into the simple formulae, Eq. [12]. Considering the above studies under weak evaporation conditions $(S \rightarrow 0), T_{\mathrm{g}} \approx T$, Eq. [12] for weak evaporation becomes

$$
\dot{n}_{\text {weak }}=\frac{1.66\left(p^{\circ}-p\right)}{\sqrt{2 \pi M R T}}
$$

The rate of intensive evaporation relative to the maximum rate of evaporation $\left(\frac{\dot{n}_{\text {intensive }}}{\dot{n}_{\text {Max }}}\right)$ has been studied in the literature. For instance, evaporation flux ratios $0.92,^{[17]} 0.82,^{[9,18]} 0.85,^{[19]}$ and 0.8 to $0.85^{[16]}$ have been reported. The reason for the smaller evaporation flux than the maximum attainable can be the backscattering of some of the particles that leave the surface. ${ }^{[9]}$

\section{VAPOR VELOCITY}

To study vacuum evaporation processes, when the condensing surface is far from the evaporating surface, we consider a pure liquid substance at constant temperature $T$ in equilibrium with its vapor in a wide and long cylinder with a frictionless piston (Figure 2(a)). The

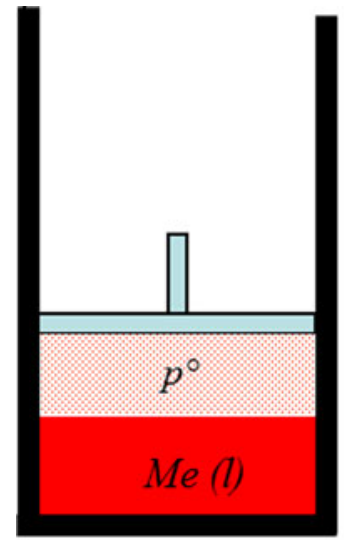

(a)

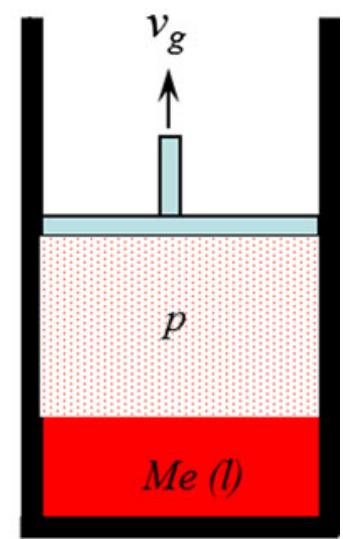

(b)
Fig. 2-Schematic of (a) equilibrium of a liquid with its vapor in a long cylinder, $(b)$ constant molar flux of liquid with its vapor under constant piston velocity. piston starts to move away from the liquid with a constant velocity of $v_{\mathrm{g}}$ (Figure $2(\mathrm{~b})$ ). The piston constant velocity causes a continuous pressure drop above the melt and simultaneous mass transfer from the liquid to the gas phase. A constant pressure $p$ is reached after a while. Then, the gas velocity above the melt and along the cylinder is constant $\left(v_{\mathrm{g}}\right)$ at pressure $p$ and vapor temperature $T_{\mathrm{g}}\left(T_{\mathrm{g}}<T\right)$, which also gives a constant molar flux. Under steady state conditions, the temperature at the liquid surface is constant and lower than the temperature of the bulk liquid due to the endothermic evaporation reaction at the surface. Here, we assume that there is no temperature gradient in the melt, which is a fair approximation, if we have a small latent heat of evaporation or rapid stirring of a large amount of liquid, while sufficient heat is given to the system to keep the liquid at constant temperature. The vapors of most metals are monatomic with close to ideal gas behavior. Thus, the molar flux in the gas phase in steady state conditions is

$$
\dot{n}_{\mathrm{g}}=\frac{v_{\mathrm{g}}}{R T_{\mathrm{g}}} p
$$

With substitution of the dimensionless external speed ratio $S$ from Eq. [6] in Eq. [14], we obtain

$$
\dot{n}_{\mathrm{g}}=S \sqrt{\frac{2}{M R T_{\mathrm{g}}}} p
$$

Assuming negligible accumulation of the atoms in the melt/gas interface and in the gas phase, we have $\dot{n}_{\text {evap. }}=\dot{n}_{\mathrm{g}}$. Then, from Eqs. [12] and [15]

$$
\left(1+\frac{2 \sqrt{\pi} S}{\eta}\right) \sqrt{\frac{T}{T_{\mathrm{g}}}}=\frac{p^{\circ}}{p}
$$

Similarly, setting $\dot{n}_{\text {evap. }}$ in Eqs. [4], [7], and [10] equal to $\dot{n}_{\mathrm{g}}$, the following relationships between $S$ and $p^{\circ} / p$ ratio are obtained from Schrage, ${ }^{[4]}$ Ytrehus and Østmo, ${ }^{[9]}$ and Algie ${ }^{[12]}$

$$
\begin{gathered}
(\Gamma+2 \sqrt{\pi} S) \sqrt{\frac{T}{T_{\mathrm{g}}}}=\frac{p^{\circ}}{p} \\
(\Gamma \beta+2 \sqrt{\pi} S) \sqrt{\frac{T}{T_{\mathrm{g}}}}=\frac{p^{\circ}}{p} \\
2 \sqrt{\pi} S \sqrt{\frac{T}{T_{\mathrm{g}}}} \cdot \frac{1+\alpha\left(\frac{\theta}{2 S}-1\right)}{\alpha}=\frac{p^{\circ}}{p}
\end{gathered}
$$

Figure 3 shows the relations between $S$ and $p / p^{\circ}$ ratio obtained from Eqs. [16] to [19]. Calculations for Eq. [16] were made for $\eta$ equal to 1.0 and 1.66 and calculations for Eq. [19] made for $\alpha$ values smaller than unity. In addition, the previously calculated curves by Ytrehus 


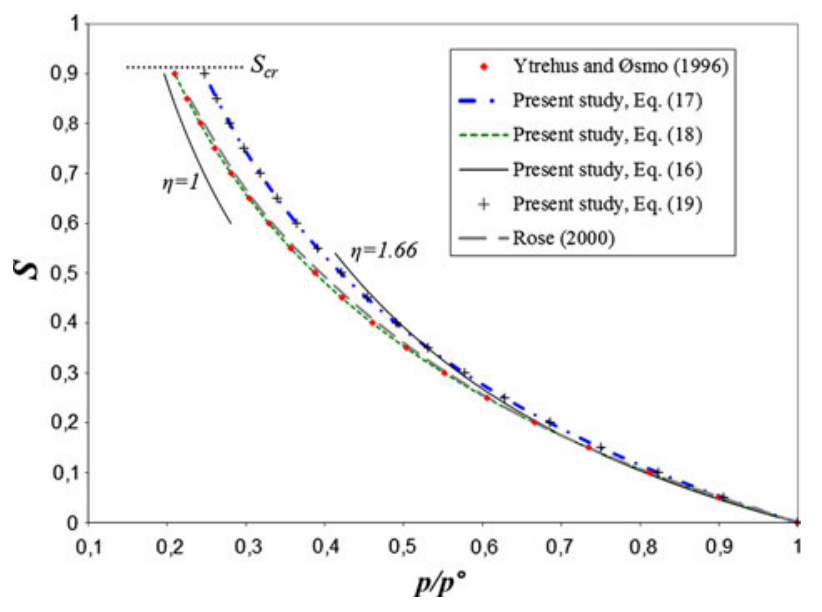

Fig. 3-Relationship between the external speed ratio $S$ and $p / p^{\circ}$ ratio determined by Eqs. [16] to [19] and the literature.

and Østmo ${ }^{[9]}$ and Rose ${ }^{[20]}$ are included in this figure. It is worth noting that Rose used a modified form of the Schrage function for the distribution of vapor molecules adjacent to the surface. As observed, for weak evaporation $\left(p / p^{\circ}\right.$ values close to unity), gas velocities obtained from Eq. [17], Eq. [18], Eq. [16] for $\eta=1.66$ and Eq. [19] for $\alpha=1$ are nearly the same. For intensive evaporation at small $p / p^{\circ}$ values, Eq. [16] for $\eta=1$ is relatively close to the results by Ytrehus and Østmo ${ }^{[9]}$ and Rose. ${ }^{[20]}$ Figure 3 shows that Eq. [18] gives a curve very near that being given by Ytrehus and Østmo. The above-given equations can be used for calculating the vapor velocities in vacuum evaporation.

\section{EFFECTIVE VACUUM PRESSURE}

According to the above model, lower vacuum chamber pressures are obtained with higher piston velocities, determined by the pumping power of the vacuum system. Equations [16] to [19] are valid for pressures in a range of $p_{\text {eff }}<p<p^{\circ}$. In pressures lower than $p_{\text {eff, }}$, the velocities exceed the attainable velocity of the vapor. The velocity limit of the vapor is considered to be the speed of sound ${ }^{[5-9]}$ in which the Mach number is unity (Mach $\left.=v_{\mathrm{g}} / c=1\right)$. The velocity of sound is expressed as

$$
c=\sqrt{\gamma \frac{R T_{\mathrm{g}}}{M}} \quad(\text { Mach No. }=1)
$$

where $\gamma$ is the ratio of specific heats and for monatomic vapor such as for the vapor of metals, it is $\gamma=\frac{C_{\mathrm{p}}}{C_{\mathrm{v}}}=\frac{5}{3} \cdot{ }^{8]}$ Substitution of $c$ instead of $v_{\mathrm{g}}$ into Eq. [6] gives a critical external speed ratio of $S_{\mathrm{cr}}=0.913$. A critical temperature ratio $\left(\sqrt{\frac{T_{\mathrm{g}}}{T}}\right)_{\mathrm{cr}}=0.818$ corresponds to this critical gas velocity, which gives $T_{\mathrm{g}}=0.67 T$. The effective vacuum pressure is determined putting these critical values into Eq. [16]

$$
p_{\text {eff }}=\frac{1}{1.22+\frac{3.95}{\eta}} p^{\circ}
$$

It is a fair approximation to take the evaporation coefficient $\eta$ as unity for pressures relatively lower than $p^{\circ}$ which yields $p_{\text {eff }}=0.193 p^{\circ} . S_{\text {cr }}=0.913$ gives effective vacuum pressures equal to $0.243 p^{\circ}$ and $0.207 p^{\circ}$ from Eqs. [17] and [18], respectively. Effective vacuum pressures $0.244 p^{\circ}, 0.22 p^{\circ}$, and $0.197 p^{\circ}$ are obtained from Eq. [19] for $\alpha=1,0.9,0.8$, respectively. Ytrehus and $\varnothing_{\text {stmo }}{ }^{[9]}$ gave $p=0.21 p^{\circ}$ as the critical pressure when the Mach number is unity. As we see, the effective vacuum pressures obtained using evaporation theories are in a narrow range of $0.19 p^{\circ}$ to $0.24 p^{\circ}$. According to Eq. [21], there is a decrease in the effective vacuum pressure with decreasing of the evaporation coefficient.

The obtained $p_{\text {eff }}$ in this study is close to that determined by Ytrehus and Østmo using another approach. The advantage of Eq. [21] here for determining $p_{\text {eff }}$ is that it distinguishes between a molecule's emission from the surface and its entry into the vapor phase by the parameter $\eta$. In order to indicate the importance of considering the evaporation coefficient, the extension of this approach for studying vacuum evaporation from multicomponent systems can be mentioned. For these systems, the physico-chemical properties of the melt components at the evaporating surface are different compared to the pure substances, and therefore different evaporation fluxes are expected. The evaporation coefficient $\eta$ can help us to consider the effect of such physico-chemical properties in the melt. Hence, the theories which consider the properties of the condensed phase such as the Algie approach and the present study are beneficial.

\section{EVAPORATION FLUX}

A dimensionless evaporation flux similar to Eq. [10] can be calculated by dividing Eq. [12] by the maximum evaporation flux $\dot{n}_{\text {Max }}$ which yields

$$
\Phi_{\mathrm{HK}}=\eta\left(1-\frac{p}{p^{\circ}} \sqrt{\frac{T}{T_{\mathrm{g}}}}\right)
$$

Similar dimensionless evaporation fluxes can be obtained considering Eqs. [4] and [7]. The relationship between $\Phi$ values and the corresponding $S$ values is shown in Figure 4 for the above-mentioned theories. The curve for $\Phi_{\mathrm{HK}}$ has been calculated for $\eta=1$. The calculated curve for the Algie approach in Figure 4 is for the case of $\alpha=1$. Although there is some agreement between all the results for weak evaporation $(S \rightarrow 0)$, differences are observed under intensive evaporation conditions. A good agreement is observed between the curves employing the Schrage and Algie approaches. A reasonable agreement is seen between the curve given by Eq. [22] and the results of Ytrehus and Østmo when the temperature difference between the two phases is considered. According to Figure 4, the Schrage and Algie approaches give the maximum evaporation rates as $0.972 \dot{n}_{\text {Max }}$ and $0.966 \dot{n}_{\text {Max }}$, respectively. The maximum evaporation rate obtained by Eq. [22] is $0.844 \dot{n}_{\mathrm{Max}}$, which is close to the calculated maximum evaporation rates in the literature mentioned above. ${ }^{[9,16,18,19]}$ Algie $^{[21]}$ presented a quantitative theory for the evaporation from 
finite surface into a vacuum and stated that the net evaporation rate is equal to the rate of phase transition only if the characteristic dimension of the surface is less than about half the mean-free path in the corresponding equilibrium vapor or if a condensing surface is placed very close to the evaporating surface. According to Algie, when the characteristic dimension of the surface is much larger than about half the mean-free path, the evaporation rate of $0.838 \dot{n}_{\mathrm{Max}}$ is obtained for $\eta=1$, which is in agreement with the present study's result. In conclusion, we may argue that approximately 84.4 pct of the number of the emitted molecules from the surface leaves the surface, and 15.6 pct of them return to the surface and condense.

Experimental data from the literature for the evaporation of pure metals $\mathrm{Zn}, \mathrm{Fe}, \mathrm{Ag}$, and $\mathrm{Si}$ are shown in Figure 4. The $S$ values corresponding to the measured $p / p^{\circ}$ values ${ }^{[22-25]}$ were determined by Eq. [16] for $\eta=1.0$ and by Eq. [18]. Although the points related to the evaporation of zinc are scattered, they show some agreement with the theoretic approaches in weak evaporation. Under intensive evaporation conditions, the $\mathrm{Zn}$ evaporation rate is much lower than that given by theory. The reason is as stated by Clair and Spenlove ${ }^{[22]}$ that the surface temperature during rapid evaporation is much lower than the average temperature of the metal due to the large amount of the heat extracted from the surface by the evaporating molecules. In addition, in their experimental design, a cylindrical cooling surface parallel to the crucible wall was located above the crucible which causes rapid temperature drop shortly above the melt, and it provides zinc deposition on the condenser surface. This is supported by the observation of condensed zinc droplets on the condensation surface by Clair and Spendlove. ${ }^{[22]}$ Hence, the lower $\mathrm{Zn}$ evaporation rates than theory in their experiments can also be attributed to the partial return of these condensed fine zinc particles to the melt. In principle, higher $\mathrm{Zn}$ evaporation rates are expected through installation of the condenser surface in a different way. The mentioned theories and present calculations in this study consider only the evaporation phenomenon and not the mass

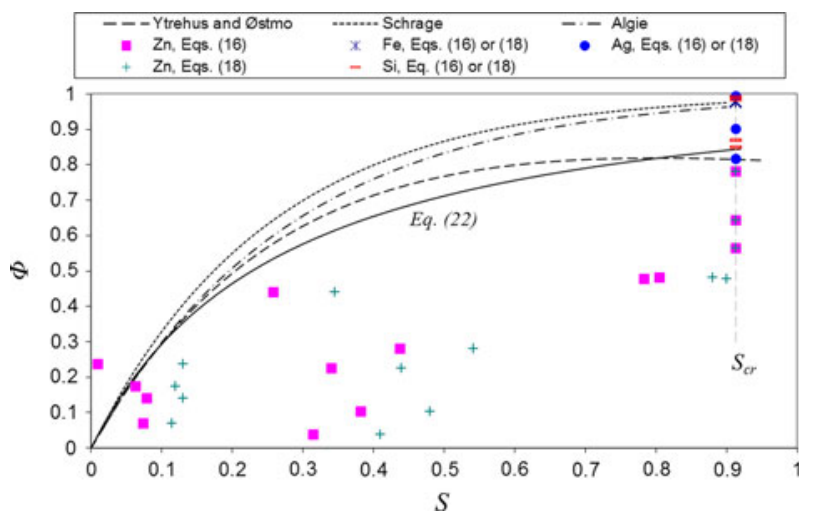

Fig. 4-Relationship between $\Phi$ and $S$ obtained in slightly different theoretic approaches, and some experimental data on the vacuum evaporation of liquid metals. Zn data are from unit values; only bulk melt temperature is measured. transfer back to the condensed phase. Although the selection of the $\mathrm{Zn}$ evaporation data ${ }^{[22]}$ may not be adequate here, it shows that the design of the experimental set-up for studying the evaporation of materials is very important and there are specific requirements with physical explanations to be considered to obtain reliable results. Figure 4 shows that there is good agreement between $\mathrm{Fe}, \mathrm{Ag}$, and $\mathrm{Si}$ evaporation and the theoretic approaches under intensive evaporation conditions. The surface temperature was measured with high accuracy in these experiments. Moreover, the condensing surface was less than the mean-free path from the evaporating surface, and no mass transfer back to the melt is expected regarding the experimental set-ups.

\section{EVAPORATION RATE OF PURE METALS}

The evaporation rate ( $\dot{n}_{\text {evap. }}$ ) by Eq. [12] for $\eta=1 v s$ the experimentally measured evaporation rates $\left(\dot{n}_{\exp }\right)$ of liquid metals is shown in Figure 5. The $\dot{n}_{\text {evap. }}$ values were calculated employing the reported standard vapor pressures for pure substances. ${ }^{[3]}$ Considering the studies by Clair and Spenlove ${ }^{[22]}$ on $\mathrm{Zn}$ evaporation, it is found that there is agreement between $\dot{n}_{\text {evap. }}$ given by Eq. [12] and $\dot{n}_{\text {exp }}$ for the experiments below $p_{\text {eff }}=0.2 p^{\circ}$. A close agreement between $\dot{n}_{\text {evap. }}$ and $\dot{n}_{\text {exp }}$ for the evaporation of $\mathrm{Fe}^{[23]}$ and $\mathrm{Ag}^{[24]}$ is observed in which the applied pressures are less than the effective vacuum pressure. Also, the temperature measurements are reliable. A majority of the measurements on the evaporation rates of $\mathrm{Si}, \mathrm{Ni}$, and $\mathrm{Ti}$ in the electron beam vacuum furnace ${ }^{[25]}$ are greater than the calculated theoretic rates. The temperatures are probably higher than those predicted in the calculations of the temperature.

Two parameters may cause a slower evaporation rate of a liquid metal than the theory: surface temperature and impurities. As mentioned before, the melt surface temperature is reduced due to the heat losses from the

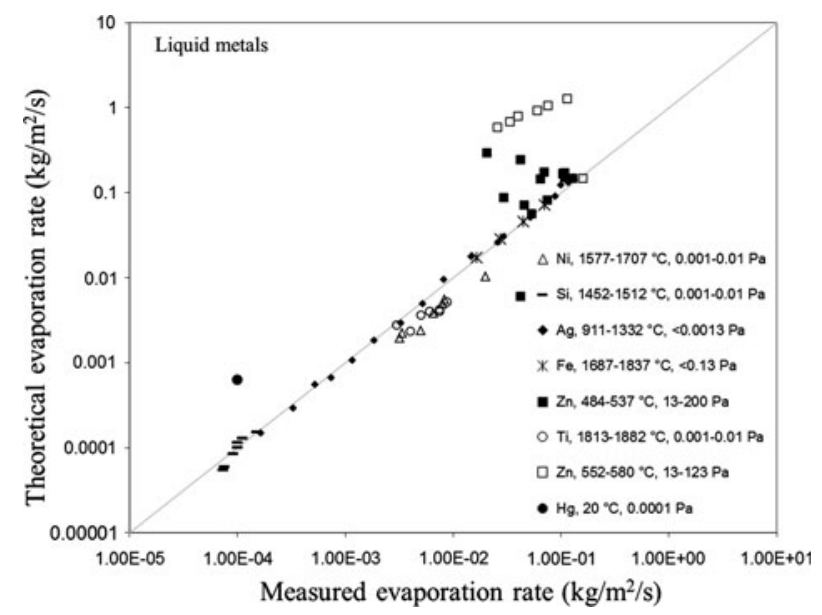

Fig. 5-The evaporation rates of liquid metals by the Hertz-Knudsen equation $(\eta=1) v s$ the experimental measurements in the literature. ${ }^{[2-25]} \mathrm{Zn}$ data are from unit values; only bulk melt temperature is measured. 


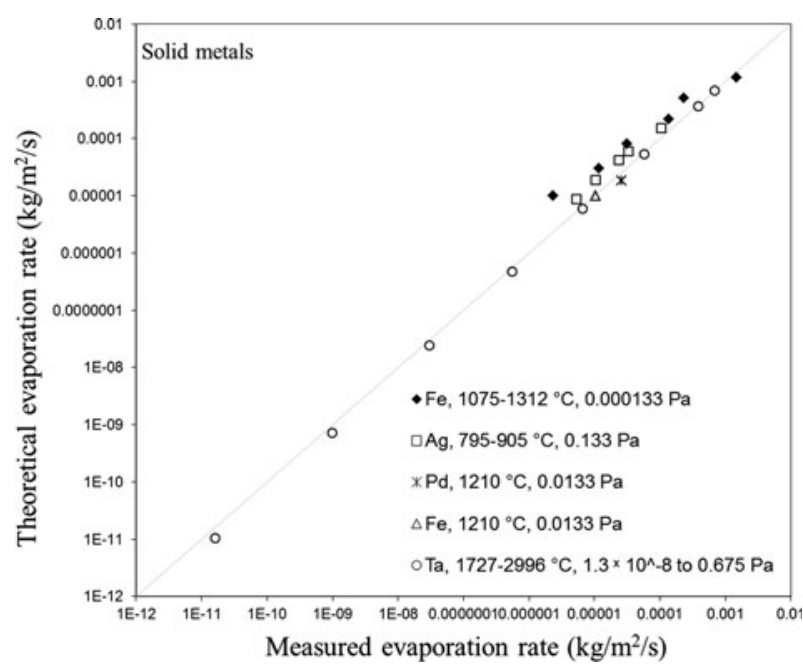

Fig. 6-The evaporation rates of solid metals by the Hertz-Knudsen equation $(\eta=1)$ vs the experimental measurements in the literature. $^{[26-30]}$

surface and the endothermic evaporation reaction at the surface. Clair and Spenlove ${ }^{[22]}$ indicated that the surface temperature in vacuum evaporation can be significantly lower than the bulk melt. This temperature difference is a very important parameter that has to be taken into account since temperature in the bulk melt is usually measured. Impurities on the melt surface in the form of solid particles or dissolved elements may influence the evaporation rate by affecting the physico-chemical properties of the liquid surface.

The theories presented here can in general be extended to the vaporization of solid metals. However, the evaporation rate is affected by the crystallographic faults and the surface atomic structure. ${ }^{[26,27]}$ The theoretic evaporation rates of selected solid metals against the measured evaporation rates are shown in Figure 6. The solid Fe evaporation measurements in high vacuum by Tsuchiyama and Fujimoto ${ }^{[28]}$ show slightly slower rates than the theoretic rates ( symbols). But, the measurement by Xiong and Hewins ( $\Delta$ symbol) is close to the theoretic value. ${ }^{[29]}$ Lowe $^{[27]}$ observed that the evaporation rate of solid $\mathrm{Ag}$ is influenced by the surface crystallographic planes at elevated temperatures. Lowe determined the evaporation coefficients as $0.8,0.85$, and 0.9 for the evaporation from (111) plane, complex plane crystal, and random polycrystal, respectively. ${ }^{[27]}$ The data presented for Ta evaporation in Figure 6 are from the measurements performed by Langmuir and Malter ${ }^{[30]}$ at high temperatures and high vacuum conditions and below the melting point of Ta. At very low pressures, higher Ta evaporation rates than the theoretic rates are observed.

\section{CONCLUSIONS}

Vacuum evaporation of pure substances is studied comparing the Hertz-Knudsen model and four newer theories. Expressions for the velocity of vapor in the vacuum evaporation process are derived. It is indicated that the vapor velocity can be calculated for both weak and intensive evaporation through consideration of the evaporation coefficient in the Hertz-Knudsen equation. An effective vacuum pressure as a function of saturated vapor pressure and evaporation coefficient, Eq. [21], is presented. The rate of evaporation should not be affected by decreasing vacuum pressure below this pressure. The experimental data on the vacuum evaporation of a majority of liquid and solid metals at low pressures with the condensing surface close to the evaporating surface are in agreement with the HertzKnudsen formula, Eq. [12] with evaporation coefficient unity.

\section{ACKNOWLEDGMENT}

The authors acknowledge the funding provided through the BASIC project (191285/V30) by the Norwegian Research Council. The authors also acknowledge Professor Leiv Kolbeinsen and Professor Merete Tangstad at NTNU for their support and advice for the present work.

\section{OPEN ACCESS}

This article is distributed under the terms of the Creative Commons Attribution License which permits any use, distribution, and reproduction in any medium, provided the original author(s) and the source are credited.

\section{REFERENCES}

1. J. Safarian and M. Tangstad: High Temp. Mater. Proc., 2012, vol. 31, pp. 73-81.

2. S. Dushman and J.M. Lafferty: Scientific Foundations of Vacuum Technique, 2nd ed., Wiley, New York, 1962.

3. O. Kubaschewski and C.B. Alcock: Metallurgical Thermo-Chemistry, 5th ed., Pergamon Press Ltd., New York, 1979.

4. R.W. Schrage: A Theoretical Study of Interphase Mass Transfer, Columbia University, New York, 1953.

5. D. Sibold and H.M. Urbassek: Phys. Fluids A, 1993, vol. 5, pp. $243-56$.

6. M.N. Kogan and N.K. Makashev: Fluid Dyn., 1971, vol. 9, pp. 913-20.

7. R. Kelly and R.W. Dreyfus: Surf. Sci., 1988, vol. 198, pp. 263-76.

8. I.J. Ford and T.-L. Lee: J. Phys. D, 2001, vol. 34, pp. 413-17.

9. T. Ytrehus and S. Østmo: Int. J. Multiph. Flow, 1996, vol. 22, pp. 133-55.

10. C. Cercignani: in Rarified Gas Dynamics, Part 1, S.S. Fisher, ed., IAA, New York, 1981, pp. 305-20.

11. T. DebRoy, S. Basu, and K. Mundra: J. Appl. Phys., 1991, vol. 70, pp. 1313-19.

12. S.H. Algie: J. Chem. Phys., 1978, vol. 69 (2), pp. 538-43.

13. P.D. Crout: J Math. Phys., 1936, vol. 15, pp. 1-54.

14. L.D. Koffman, M.S. Plesset, and L. Lees: Phys. Fluids, 1984, vol. 27, pp. 876-80.

15. T.M. Muratova and D.A. Labuntsov: High Temp., 1969, vol. 7, pp. 888-96.

16. D.A. Labuntsov and A.P. Kryukov: Int. J. Heat Mass Transf., 1979, vol. 22, pp. 989-1002.

17. N.K. Makashev: Fluid Dyn., 1972, vol. 5, pp. 130-38.

18. F.G. Gheremisin: Izv. Akad. Nauk SSSR, Mekh. Zhidk. Gaza, 1972, vol. 2, pp. 176-78.

19. J. Fischer: Phys. Fluids, 1976, vol. 19, pp. 1305-11.

20. J. Rose: Int. J. Heat Mass Transf., 2000, vol. 43, pp. 3869-75. 
21. S.H. Algie: Vacuum, 1976, vol. 26, pp. 503-10.

22. H.W. St Clair and M.J. Spenlove: Trans. AIME, 1951, vol. 191, pp. 1192-97.

23. P.N. Smith and R.G. Ward: Can. Metall. Q., 1966, vol. 5, pp. 77-92.

24. C.T. Ewing and K.H. Siern: J. Phys. Chem., 1975, vol. 79, pp. 2007-17.

25. Y. Ogasawara, T.S. Tabaian, and M. Maeda: ISIJ Int., 1998, vol. 38, pp. 789-93.
26. J.P. Hirth and G.M. Pound: Acta Metall., 1957, vol. 5, pp. 649-53.

27. R.M. Lowe: Acta Metall., 1964, vol. 12, pp. 1111-18.

28. A. Tsuchiyama and S. Fujimoto: Proc. NIPR Symp. Antarct. Meteorites, 1995, vol. 8, pp. 205-13.

29. Y. Xiong and R. Hewins: 63rd Annual Meteoritical Society Meeting, 28 Aug-1 Sep 2000, Chicago, 5089.

30. D.B. Langmuir and L. Malter: Phys. Rev., 1939, vol. 55, pp. $748-49$. 